\section{INSTITUCIONES, INNOVACIÓN $Y$ TRANSFERENCIA \\ DE CONOCIMIENTO: \\ CONTRIBUCIONES DE LOS \\ ESTUDIOS SOBRE LAS \\ VARIEDADES DEL CAPITALISMO'}

\author{
Hugo Pinto \\ Centro de Estudios Sociales \\ Universidad de Coimbra
}
INSTITUTIONS, INNOVATION AND KNOWLEDGE TRANSFER: CONTRIBUTIONS FROM THE PERPECTIVES ON THE VARIETIES OF CAPITALISM

\begin{abstract}
This article is an interpretation of the theories about the varieties of capitalism, highlighting their implications for innovation, especially for the processes of knowledge transfer between universities and businesses. Studies of the varieties of capitalism point to the importance of the institutional architecture for the competitiveness of countries. The debate highlights the institutional complementarities as a useful concept in theoretical terms to explain the processes of development and need to anchor institutional analysis on empirical data. These approaches are relevant to frame important theoretical studies on innovation, where knowledge transfer processes are considered one of the essential impetus for innovation. The article provides a critical review of three approaches to varieties of capitalism: the pioneering study of Hall and Soskice, the business systems of Whitley and the approach to governance of Amable and Boyer. Corollaries that can connect to processes of knowledge transfer are extracted for each approach. The conclusions indicate that the focus of attention to the company should be complemented with a systemic approach, to show the wide range of actors, where the university becomes one of the most important. Government intervention is essential in shaping an innovative behaviour of the actors, to encourage and promote the use of different mechanisms of knowledge transfer.
\end{abstract}

KEY WORDS: Knowledge transfer; innovation; institutions; varieties of capitalism; social systems of innovation and production.

\section{INTRODUCCIÓN}

Hace una década Hall y Soskice (H\&S, 2001) introdujeron el concepto de las variedades del capitalismo en el análisis de la diversidad institucional de los paises. En este texto seminal muestran que la especificidad y la complementariedad de las arquitecturas institucionales se reflejan en
RESUMEN: Este trabajo realiza una interpretación de las teorías sobre las variedades del capitalismo resaltando sus implicaciones para la innovación, en especial para los procesos de transferencia de conocimiento entre la universidad y la empresa. Los estudios de las variedades del capitalismo señalan la importancia de la arquitectura institucional para la competitividad de los países. El debate pone de relieve las complementariedades institucionales como concepto útil en términos teóricos para explicar los procesos de desarroIlo, así como necesidad de anclar el análisis institucional en datos empíricos. Estos enfoques son un complemento teórico importante para enmarcar los estudios sobre innovación, donde los procesos de transferencia de conocimiento se consideren uno de los aspectos esenciales del impulso de las innovaciones. El artículo realiza una revisión crítica de tres enfoques sobre variedades de capitalismo: el estudio precursor de Hall y Soskice, el de los sistemas empresariales de Whitley y el enfoque de la gobernanza de Amable y Boyer. Para cada uno de ellos se extraen corolarios que permiten conectarlos con los procesos de transferencia de conocimiento. El resultado señala que el foco central de atención a la empresa debe complementarse con un enfoque sistémico que permite ver la amplia gama de actores, donde la universidad se convierte en uno de los más relevantes. La intervención del gobierno es esencial en la configuración de un comportamiento innovador de los actores, para fomentar y promover el uso de diferentes mecanismos de transferencia de conocimiento.

PALABRAS CLAVE: Transferencia de conocimiento; innovación; instituciones; variedades de capitalismo; sistemas sociales de innovación y producción.

las diferentes capacidades de adaptación de los estadosnación a los desafíos de la globalización. Los paises no convergen hacia un único modelo de capitalismo neoliberal. Al contrario, las ventajas comparativas institucionales de cada tipo de arquitectura instigan diferentes respuestas a la globalización económica. Como puso de relieve Hancké (2009), estos enfoques se consideran una de las innovacio- 
nes más importantes de los estudios comparativos en las ciencias sociales, habiéndose convertido con rapidez en una de las teorías centrales de la actual economía política.

Una de sus potencialidades se encuentra en su utilidad para entender los procesos de innovación, en particular las políticas de innovación que intentan dinamizar los sectores productivos facilitando los procesos de transferencia de conocimiento. En estos procesos las organizaciones universitarias se consideran actores estratégicos para potenciar las innovaciones. Un ejemplo relevante lo encontramos en la Unión Europea. La Agenda de Lisboa sitúa a la transferencia de conocimiento como un tema crucial para los estados miembros, haciendo especial hincapié en el papel que las universidades pueden desempeñar en la competitividad y la innovación. Se sostiene que, más allá de la investigación y la educación tradicional, la universidad también debe desarrollar actividades de transferencia de conocimiento que tengan consecuencias para el desarrollo territorial. Sin embargo, las políticas de crecimiento basadas en la innovación se encuentran con el dilema de que el alcance de este proceso de transferencia suele depender en gran medida de la diversidad de entornos institucionales.

En la literatura especializada sobre innovación se considera a las instituciones como un elemento importante, aunque éstas resultan difíciles de contextualizar y ubicar en los elementos que conforman el sistema económico y social que caracteriza a los paises. Los estudios que contemplan los rasgos del capitalismo en estados y regiones suponen una fuente relevante que puede enriquecer las investigaciones sobre innovación, en particular los factores estructurales que afectan a los procesos de transferencia de conocimiento en el sistema público de I+D.

Este artículo aborda tres ramas de las variedades del capitalismo con el objetivo de encontrar pistas acerca de cómo estas teorías afectan a la transferencia de conocimiento: las ideas fundadoras de H\&S, los sistemas empresariales de Whitley (1999) y la visión inspirada por la escuela francesa de la regulación de sistemas sociales de la innovación y la producción (SSIP). El objetivo es ilustrar cómo el entorno institucional afecta a procesos centrales para la actividad innovadora.

La estructura del artículo es la siguiente. Después de la introducción, en el punto 2 se exponen las ideas claves sobre innovación que guian el actual debate, seguido de las principales asunciones sobre el papel de la universidad en los procesos de innovación. El punto 3 se dedica a analizar los tres enfoques teóricos sobre las variedades del capitalismo. Para cada uno de ellos se resaltan sus principales asunciones y tipologías, estableciendo unas conclusiones a modo de corolario referidas a sus implicaciones para interpretar los procesos de transferencia de conocimiento. En el punto 4 se ofrecen unas conclusiones críticas que resaltan la utilidad de estos enfoques para estudiar la capacidad innovadora de los países de forma comparada, así como las limitaciones que aún existen en este campo.

\section{INNOVACIÓN, UNIVERSIDADES Y TRANSFERENCIA DE CONOCIMIENTO}

\subsection{Ideas preliminares sobre la innovación y la transferencia de conocimiento}

El concepto de innovación ha evolucionado en los últimos cincuenta años desde un enfoque centrado en la tecnología hacia otro que destaca la amplia complejidad y variedad de dimensiones, escalas y actores (OCDE, 2005), aunque estos estudios siguen siendo deudores de la inspiración schumpeteriana que considera a la innovación principalmente como un producto o proceso nuevo o mejorado. Esta visión no suele ser muy eficaz en términos de políticas operativas. No obstante, dejar abierto el concepto de innovación permite una mejor comprensión de que la innovación es el resultado de vías específicas donde la tecnología y el conocimiento se desarrollan en la interacción de múltiples factores determinados contextualmente. Las influencias que tienen la demanda del mercado y la comercialización en la decisión de producción de conocimientos son ejemplos de dependencias que llevan a algunas empresas, regiones o paises a caminos y trayectorias especificas. La aparición del enfoque sistémico de la innovación ha aumentado la atención que se presta al componente institucional en los procesos de creación, transferencia y aplicación del conocimiento, haciendo ahínco en las condiciones, reglamentos y políticas en las que operan los mercados, así como en el papel del gobierno en este contexto. La centralidad otorgada a la innovación pone de relieve la importancia de los conocimientos científicos para el progreso económico. En consecuencia, se otorga un papel más relevante 
a las universidades, en comparación con el punto de vista más tradicional de la innovación, en el que la empresa era el objeto principal de atención. Éste es uno de los presupuestos de las teorias que ponen de relieve el nuevo papel del conocimiento, que destaca a la universidad como organización integrada en la creación de dinámicas locales de desarrollo a través de la investigación básica y aplicada. En este contexto es especialmente importante analizar la transferencia de conocimiento como un aspecto central de la competitividad y de la cohesión de los territorios, en particular la estructura de las interconexiones entre los actores de los sistemas de innovación.

La transferencia de conocimiento ha sido objeto de varios enfoques influentes en los últimos años. Este artículo se centra en la transferencia de conocimiento que realiza la universidad, considerada como un productor y protector de los bienes comunes que son resultado de la investigación científica, tanto para la sociedad que la rodea como para las empresas que absorben estos conocimientos y los Ilevan al mercado. Este concepto recoge numerosos elementos que se centran en la producción académica del conocimiento científico, en su aplicación en la empresa y en el logro de potencial económico. Aunque el concepto originalmente utilizado por muchos autores en este campo es el de "transferencia de tecnología", aqui se ha optado por hablar de la transferencia de conocimiento debido a que este concepto engloba dimensiones adicionales y parece más adecuado frente a la dificultad que supone separar los mundos de la ciencia, la tecnología y la innovación (Latour 1987, 2005).

La formulación de políticas adecuadas para fomentar la transferencia de conocimiento a las particularidades de cada situación requiere una comprensión de las especificidades del territorio, en particular las instituciones relacionadas con las actividades innovadoras. La mera repetición de buenas prácticas de transferencia de conocimiento, como suele realizarse en gran parte de las políticas de innovación, no es suficiente para alcanzar logros de desarrollo. Volviendo al ámbito de la Unión Europea, los contextos institucionales en que se aplican las políticas son en ocasiones tan diferentes que los resultados de replicar buenas prácticas resultan frecuentemente en fracasos. La ambición de convertir la Unión Europea en un espacio competitivo se enfrenta a las dificultades provenientes de la falta de cohesión. Es extremadamente difícil responder adecuadamente a los desafíos de la diversidad de las arquitecturas institucionales, los incentivos y el comportamiento de los actores en el ámbito de la innovación.

\subsection{El cambio en la Universidad y la importancia de la transferencia de conocimiento}

Las universidades son las organizaciones más relevantes en la producción de conocimientos, asumiendo además el papel de nodos en las redes cientificas y tecnológicas mundiales (David, 1994). Actualmente las universidades están experimentando una importante transformación marcada por un cambio de paradigma. Además del papel tradicional de generación de conocimientos, se espera de ellas que respondan a una nueva gama de necesidades sociales.

Un grupo de autores encabezados por Michael Gibbons (Gibbons et al., 1994) han introducido la noción de un sistema de investigación nuevo, más interactivo y socialmente distribuido, llamado Modo 2. Sostienen que la producción de conocimiento, que en el pasado estaba estructurada en las disciplinas cientificas universitarias, es ahora mucho más heterogénea. El nuevo modo de producción de conocimiento no sustituye por completo a su predecesor, el Ilamado Modo 1, sino que es su complemento. El conocimiento en el Modo 2, que Boaventura de Sousa Santos (2008) designa como "conocimiento pluriuniversitario", se genera en un contexto de mayor alcance, en esencia transdisciplinaria, tratando de movilizar a las perspectivas teóricas y metodologías empíricas para resolver problemas específicos. En el Modo 2, los actores involucrados son más numerosos y tienen una interacción más intensa. Una caracteristica muy importante se refiere a la reflexividad creciente del proceso de creación de conocimiento, un proceso dialógico capaz de incorporar una mayor diversidad de puntos de vista. Esto significa que a los sistemas de control de calidad científica, como a la supervisión de los pares, se añaden otros criterios de complejidad económica, social y cultural para identificar lo que se considera como buena ciencia.

Este conocimiento pluriuniversitário es, según Boaventura de Sousa Santos (Ibíd., 35), un conocimiento del contexto, que actúa como principio organizador de su producción y su aplicación, lo que ocurre en la mayoría de los casos fuera del ámbito universitario. Los criterios de relevancia se derivan, por tanto, de nociones procedentes tanto de

ARBOR Vol. 188753 enero-febrero [2012] 31-47 ISSN: 0210-1963

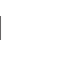


investigadores como de usuarios, lo que conlleva un carácter más transdisciplinar. El nuevo modo de producir conocimiento resulta más evidente en las asociaciones universidad-industria, así como en el conocimiento producido directamente para el mercado, aunque que no se agota en esta escala y alcanza una complementariedad que involucra a varios actores. El papel de la universidad se ha reconfigurado en el sentido de que sus conocimientos contribuyen más directamente a la competitividad económica. Las áreas de investigación se orientan por las perspectivas de ser absorbidas por las empresas y convertirse en nuevos productos y procesos. Igualmente, las restricciones en la financiación pública de las universidades han funcionado como condicionantes e incentivos para tratar de establecer asociaciones con el sector privado dirigidas a la comercialización de resultados de la investigación.

Otro de los enfoques relevantes en este ámbito es el de la Triple Hélice, el famoso modelo de Leydesdorff y Etzkowitz (1997). El modelo muestra la intersección de tres esferas y múltiples interacciones entre los diversos actores. Su papel en la dinámica del sistema resulta en un espacio compartido que surge de las necesidades de los nuevos actores capaces de una intermediación eficaz. La Triple Hélice aboga por la idea de que la nueva universidad es una universidad emprendedora (Etzkowitz, 2003, Etzkowitz et al., 2000). Ejerce una tercera misión que incluye, como destacan Gunasekara (2006) o Goldstein (2008), una serie de actividades empresariales: promover el desarrollo económico regional, fomentar y recompensar los profesores que prestan asistencia técnica a los actores en la región, estudios de mercado, contribución a la creación de empresas de base tecnológica, así como participación en las inversiones de empresas nuevas que surgen del conocimiento generado en el mundo académico.

Algunos autores han discutido y comparado la visión del Modo 2 con otros métodos relacionados con los cambios en el sistema científico (Hessels y Van Lente, 2008), mostrando cómo su amplio alcance ha generado gran atención por parte de la política científica. En el caso de los Estados Unidos, la importancia de la transferencia de conocimiento como tema central para las políticas de investigación e innovación ha aumentado desde principios de los ochenta con el Bayh-Dole Act. Berman (2008) subraya la importancia de este evento en el proceso de institucionalización, señalando el papel de las prácticas asociadas a transferencia de co- nocimiento, especialmente en los beneficios económicos de la propiedad de las patentes resultantes de la investigación financiada con fondos públicos. Los estudios en este campo muestran que los cambios en las prácticas de la universidad ya estaban en marcha, aunque se han institucionalizado más efectivamente debido a los nuevos marcos legales. En el caso de Europa, también existen ejemplos notables, como el precursor programa europeo ESPRIT centrando en tecnologías de la información, que han dado importancia a la cooperación público-privada para la investigación. El primer Programa Marco de Investigación y Desarrollo Tecnológico (1984-1987) aumentó la atención prestada a esta cuestión, siendo asumido como una referencia esencial en toda la Unión Europea. Otro hito importante fue el Libro Verde de la Innovación (Comisión Europea, 1995), donde se pone de relieve el surgimiento de la economía del conocimiento y el papel que en ella tiene la universidad como jugador clave para la generación y aplicación de conocimiento, unido a los instrumentos que facilitan la conexión entre la academia y la empresa. Adicionalmente, en la UE la importancia de la transferencia de conocimiento se ha visto reforzada por la brecha en las capacidades tecnológicas, comparadas con las realidades de los EE.UU. y Japón, que también aparecen por primera vez en el Libro Verde y se reiteran en las evaluaciones que se realizan sobre la capacidad innovadora en el ámbito europeo.

Finalmente, en el contexto del cambio de paradigma político y organizativo, existe un creciente campo de estudio que muestra la diversidad de canales y mecanismos por los que las universidades influyen en el desarrollo. Debackere y Veugelers (2005) identifican como las principales conexiones formales de la ciencia con la industria las siguientes: la creación de empresas tecnológicas, los acuerdos de colaboración en la investigación en consorcio, los contratos de investigación y consultoría y la explotación de los derechos de propiedad industrial. Otros canales también se resaltan como importantes, como la cooperación en la formación de graduados, la formación para los empleados de las empresas y los intercambios de personal entre la empresa y la universidad. Estos autores añaden que detrás de la variedad de relaciones formales existe una variada serie de contactos informales y redes personales. Aunque difíciles de medir, estos contactos informales son fundamentales para la promoción de nuevas relaciones formales. Para que surja un interés de ambas partes para establecer vínculos, es necesaria la conciliación entre la demanda y oferta de los 
conocimientos existentes, donde tiene especial importancia lo que Cohen y Levinthal (1990) definen como "capacidad de absorción" por parte de las empresas.

En la misma línea, Bercovitz y Feldman (2005) identifican cinco mecanismos formales e informales para la transferencia de conocimiento entre universidades y empresas que sintetizan estas diversas contribuciones: la investigación financiada, las licencias de patentes, la contratación de estudiantes, la creación de nuevas empresas y la "serendipidad". D'Este y Patel (2007) señalan que hay abundante evidencia empírica de que la transferencia de conocimiento se produce a través de múltiples canales, tales como la creación de nuevas infraestructuras físicas, los contratos de consultoría y investigación, los consorcios de investigación, las reuniones de capacitación y las conferencias. En consecuencia, centrar la discusión en la promoción de las patentes y la creación de empresas puede dar lugar a efectos negativos en los esfuerzos de otros canales. Estos autores destacan varios grupos de factores relevantes que influyen en la interacción entre la universidad y la industria. A saber, la orientación comercial de la universidad (que puede observarse mediante la existencia en su misión de apoyar el desarrollo regional o la parte de financiación de la investigación procedentes de la industria), las características de los departamentos (tales como la escala de los recursos, la calidad de la investigación y la estrecha colaboración con la industria) y las características individuales de los investigadores (variables relevantes son la capacidad de atraer la inversión, el estatus académico, la edad, las normas culturales de los campos científicos y la experiencia previa en la industria).

El conjunto de trabajos citados en esta sección sirve para subrayar la necesidad de un concepto amplio de trans- ferencia de conocimiento, tal como sostienen Bozeman (2000) y Molas-Gallart et al. (2002). Se trata de un concepto distintivo, e interconectado con otros como la difusión de tecnología y difusión de la innovación. La transferencia de conocimiento es un proceso voluntario de participación activa de beneficio mutuo entre las organizaciones de investigación, las empresas, los gobiernos o la comunidad más amplia. El objetivo es generar, adquirir, implementar o facilitar el acceso a los conocimientos necesarios para mejorar el bienestar material, humano, social o ambiental. La comercialización de la ciencia, por tanto, debe ser vista como un componente de los procesos de transferencia de conocimiento.

\section{LA tRANSFERENCIA DE CONOCIMIENTO y EL CONTEXTO INSTITUCIONAL}

En la sección anterior se ha destacado la importancia de la transferencia de conocimiento y se han identificado los mecanismos clave que caracterizan este proceso. La pregunta que nos planteamos a continuación es, ¿cuáles son los diversos mecanismos que generan diversidad en el comportamiento de los actores? Una respuesta aceptable podría basarse en las ideas de Phan y Siegel (2006), que discuten la efectividad de la transferencia (de la tecnología, en su sentido más estricto) centrándose en la cuestión de las licencias de patentes. Los autores muestran cuatro dimensiones clave completamente entrelazadas que afectan al comportamiento de los actores en la transferencia de conocimiento: el contexto organizativo, la eficacia de la transferencia, el contexto institucional y el contexto individual (Tabla I).

TABLA I. CONTEXTOS QUE INFLUYEN EN LA TRANSFERENCIA

\begin{tabular}{|c|l|}
\hline \multicolumn{1}{|c|}{ Contexto Organizacional } & \multicolumn{1}{c|}{ Efectividad } \\
\hline Arquitectura estructural, flujos de información, formas legales & Licenciamiento y creación de empresas \\
\hline Contexto Institucional & \multicolumn{1}{c|}{ Contexto Individual } \\
\hline Políticas, prácticas, valores comunes, sistemas de incentivos & $\begin{array}{l}\text { Ética profesional, objetivos personales, actitudes, experiencia, conocimien- } \\
\text { to y competencias }\end{array}$ \\
\hline
\end{tabular}

Fuente: Phan y Siegel (2006: 53) (adaptada por el autor). 
Estos autores señalan, por ejemplo, que las oficinas de transferencia de conocimiento en las universidades de los EE.UU., al centrar su acción en las cuestiones de la concesión de licencias, han alentado el aumento en el número de patentes. El éxito de la comercialización de la investigación académica se deriva de los incentivos individuales, la propensión al riesgo y las habilidades de los académicos y empresarios, que dependen en gran medida de la actual arquitectura institucional. Por otra parte, la importancia de adaptar el análisis institucional en el ámbito económico se justifica, de acuerdo con Jackson y Deeg (2006), debido a que las economías nacionales se caracterizan por determinadas configuraciones institucionales que afectan a la economía y tienen diferentes efectos sobre el desempeño de los países, contribuyendo a crear dependencias de trayectoria.

Sin embargo, un obstáculo siempre presente en estos enfoques reside en la dificultad inherente al concepto de institución. Los esfuerzos realizados para definir las instituciones son aún una tarea inacabada debido a que las aproximaciones son incompletas y frecuentemente incompatibles entre sí, aunque resulta útil partir de algunos rasgos comunes de las definiciones de los autores centrales del institucionalismo. Las instituciones son, según Hodgson (2006), los tipos de estructuras de poder en la sociedad, los sistemas de reglas que estructuran las interacciones sociales frecuentes. North (1994) afirma que las instituciones son limitaciones humanamente creadas para la estructura de la interacción humana, que consisten en las limitaciones formales (normas, leyes, constituciones), las restricciones informales (normas de comportamiento, convenciones y códigos auto-impuestos) y las formas de directrices que definen la estructura de incentivos en la sociedad. Aoki (2001) entiende a una institución como un patrón autosostenible de interacciones sociales, representado por las normas que todos los jugadores conocen e incorporan como creencias compartidas acerca de cómo se actúa en un determinado ámbito. Bromley (2006) insiste en que una comprensión clara de las instituciones requiere que sean consideradas tanto en su dimensión más estrecha de restricción a la conducta como en la dimensión liberadora de los individuos para definir conjuntos de oportunidades en los campos de acción para los miembros de ciertos grupos. Las instituciones se asumen de esta manera como un elemento de las relaciones económicas y sociales, y no sólo como límites a esas relaciones. Nelson (2008) ilumina el concepto al conectar a las instituciones con las tecnologías sociales. La transferencia, la difusión y la propagación de ciertas tecnologias dependen de un contexto institucional adecuado para su uso, en lo que coindicen con estudios previos, como los de Freeman y Louçã (2004) o Freeman y Pérez (1988). Finalmente, cabe destacar que Jackson y Deeg (ibíd.) sugieren varios ámbitos institucionales que deben ser comparados: los sistemas financieros, los gobiernos corporativos, las relaciones entre empresas, las relaciones laborales, la formación profesional, la creación de empleo, el estado de bienestar y la innovación.

En este marco de discusión general caracterizado por la importancia otorgada a la estructura institucional, se han seleccionado tres grupos de autores que distinguen entre diferentes ramas de las variedades de capitalismo debido a su utilidad para interpretar los procesos de transferencia (Tabla II). La primera se trata del trabajo precursor de Hall y Soskice. La segunda es la referida a los sistemas nacionales de empresa de Richard Whitley, mientras que la tercera, llamada en ocasiones de métodos de gobierno, comprende los trabajos sobre sistemas sociales de la producción de Hollingsworth y Boyer y sobre los sistemas sociales de la innovación y la producción de Amable.

\subsection{La coordinación en las economías de mercado}

El planteamiento iniciado por Hall y Soskice (2001) ha sido propuesto para explicar las similitudes de las economías desarrolladas. Ello se realiza vinculando los procesos de modernización con la presencia de modelos de producción diferentes para tratar de comprender las interacciones estratégicas, donde la empresa se sitúa en el centro de los sistemas y define su actividad. Los sistemas relevantes son: el sistema de gobierno corporativo, el sistema de relaciones entre empresas, las relaciones laborales y el sistema educativo y de formación. Se trata de un enfoque micro-analítico sobre las interacciones estratégicas de la empresa, que se entiende como "institucionalmente incorporada". La comparación entre los países se realiza observando la manera en que las empresas resuelven sus problemas de coordinación.

Estos autores introducen el concepto de ventaja comparativa institucional, a saber, una arquitectura institucional de una determinada economía política que proporciona a las empresas ventajas en actividades especificas. Con 
TABLA II. LAS COMPARACIONES DEL CAPITALISMO EN LOS MARCOS SELECCIONADOS

\begin{tabular}{|c|c|c|c|}
\hline $\begin{array}{c}\text { Autores } \\
\text { representativos }\end{array}$ & Dominios Institucionales & Grupos de Países & Notas \\
\hline Hall y Soskice & $\begin{array}{l}\text { Sistemas financieros, relaciones indus- } \\
\text { triales, competencias, coordinación inter- } \\
\text { empresa }\end{array}$ & $\begin{array}{l}\text { Economias liberales de mercado vs. } \\
\text { Economías coordinadas de mercados (in- } \\
\text { dustria vs. grupos coordenados) }\end{array}$ & Análisis de costes de transacción \\
\hline Whitley & $\begin{array}{l}\text { Estados, sistemas financieros, competen- } \\
\text { cias, confianza/autoridad }\end{array}$ & $\begin{array}{l}\text { Tipos ideales: fragmentado, proyecto en } \\
\text { red, distrito industrial coordinado, con- } \\
\text { glomerado financiero, conglomerado in- } \\
\text { tegrado, conglomerado compartimentado, } \\
\text { conglomerado colaborativo y conglome- } \\
\text { rado altamente colaborativo }\end{array}$ & $\begin{array}{l}\text { Compara dimensiones relacionadas con } \\
\text { organización horizontal vs. vertical, con- } \\
\text { trol de la propiedad y dependencia traba- } \\
\text { jador-capitalista }\end{array}$ \\
\hline $\begin{array}{l}\text { Hollingsworh, } \\
\text { Boyer, Petit, } \\
\text { Amable }\end{array}$ & $\begin{array}{l}\text { Competición de los mercados de pro- } \\
\text { ductos, instituciones del mercado labo- } \\
\text { ral, gobernanza financiera y corporativa, } \\
\text { protección social y estado de bienestar, y } \\
\text { sistema de educación y entrenamiento }\end{array}$ & $\begin{array}{l}\text { Clusters de cinco paises: capitalismo fun- } \\
\text { dado en el mercado, mesocorporativista } \\
\text { (asiático), europeo continental, socialde- } \\
\text { mócrata y mediterráneo }\end{array}$ & $\begin{array}{l}\text { Basado en seis mecanismos de goberna- } \\
\text { ción para coordinación de las transaccio- } \\
\text { nes: mercados, jerarquias, estados, aso- } \\
\text { ciaciones, redes y comunidades. Utilizan } \\
\text { métodos inductivos de agregación }\end{array}$ \\
\hline
\end{tabular}

Fuente: Jackson y Deeg (2006: 31), adaptada por el autor.

esta arquitectura, las empresas pueden realizar ciertas actividades y producir determinados bienes de manera más eficiente que otras debido al apoyo institucional. Dado que no existe una distribución similar de las instituciones por los territorios, surge una variedad de perfiles de rendimiento productivo y económico. Distinguen dos tipos básicos de regímenes de producción: las economías liberales de mercado (ELM) y las economías coordinadas de mercado (ECM). Las economías liberales de mercado comparten características como la orientación de negocio a corto plazo, los mercados de trabajo desregulados, la alta educación general y la integración en un ambiente de negocios internacional de alta competencia. En las economías coordinadas, el comportamiento económico está estratégicamente coordinado a través de otros mecanismos fuera del mercado. Existe un mayor énfasis en las finanzas a largo plazo. Los niveles de la formación profesional son altos, al igual que la cooperación tecnológica entre las empresas existentes. Las ECM tienden a concentrar sus inversiones en activos específicos y co-específicos, mientras que las ELM prefieren activos fácilmente cambiables.
Estas características permiten resaltar cómo funcionan las complementariedades institucionales. Se sostiene que dos instituciones son complementarias si la presencia (o eficiencia) de una institución mejora el rendimiento (o eficiencia) de la otra. Por ejemplo, un enfoque a corto plazo requiere de una capacidad de ajuste rápido de la mano de obra, lo que resulta necesario en un mercado laboral flexible donde se puede contratar y despedir con facilidad. Sin embargo, como sugieren estos autores, muchos paises no tienen una forma consistente del capitalismo. Es decir, en la realidad presentan versiones intermedias de los tipos ideales, dado que sus instituciones no generan complementariedades, sino potenciales ineficiencias, resultando en un desempeño económico más débil. Los patrones de especialización tecnológica están en gran parte determinados por el tipo de capitalismo predominante en un país. Las ELM tienden a especializarse en la innovación radical, mientras que las ECM se concentran en mayor medida en la innovación de naturaleza incremental. La innovación radical es particularmente relevante en sectores de rápida evolución tecnológica, que carecen de capacidad de asumir riesgos en las estrategias de mejora de productos

ARBOR Vol. 188753 enero-febrero [2012] 31-47 ISSN: 0210-1963 
y de acelerar su implementación en sectores productivos extensos. La innovación incremental, en cambio, tiende a ser más importante para mantener la competitividad en la producción de bienes de capital, en mantener la calidad del producto y en asegurar la lealtad del cliente, algo que es más coherente con relacionales estables de las ECM. En este sentido, Casper et al. (1999) muestran que las empresas de biotecnología y software en Alemania habian logrado con éxito entrar en estos sectores avanzados, pero que tenían más potencial en sectores caracterizados por la tecnología basada en el conocimiento acumulado con menos riesgo de implantación en el mercado.

\section{Corolario 1}

Las arquitecturas institucionales discutidas por Hall y Soskice ilustran algunas implicaciones relevantes para la transferencia de conocimiento. A partir de la distinción entre el énfasis en innovaciones radicales de las economías liberales y el realizado en innovaciones incrementales por las economías coordinadas, Casper (2006) pone de relieve cómo las ECM tienen una mayor coordinación en el sistema de formación, al igual que entre la industria y la academia, debido al diseño de los planes de estudio y la investigación aplicada. En los países de ELM, la relación entre las universidades y las empresas está muy centrada en I+D contratada $y$, en última instancia, se basa en transacciones de corto plazo, especialmente con la concesión de licencias de derechos de propiedad industrial (DPI) de las universidades a las empresas establecidas 0 nuevas empresas creadas y dirigidas por los investigadores. El marco legal y las oficinas de transferencia de conocimiento son los facilitadores de estos mecanismos formales. Por otra parte, las relaciones en las ECM a largo plazo son facilitadores de la investigación aplicada en los departamentos y las grandes empresas, donde los marcos legales potencian que el investigador posea el resultado de la investigación financiada con fondos públicos. En estos contextos hay menos atención a los aspectos relacionados con la comercialización y propiedad de la transferencia de conocimiento.

\subsection{Los sistemas empresariales y los tipos de estado}

El segundo tipo de enfoque sobre las variedades del capitalismo es la propuesta de Whitley $(2007 a, 1999)$ referida a los sistemas empresariales. Estos sistemas se definen como distintos patrones de organización económica que varían en grado y modo de coordinación de las actividades económicas, así como la organización y la interconexión entre los accionistas, gerentes, especialistas y demás personal. Este autor establece conexiones entre las macroinstituciones, los sistemas de innovación y los modos de organización. Señala que los arreglos institucionales limitan y facilitan el desarrollo de habilidades empresariales, en particular de la capacidad de innovación. Ciertas instituciones alientan a las empresas a desarrollar capacidades competitivas más eficaces en industrias específicas. Estas caracteristicas explican los tipos de especialización y los resultados económicos. Whitley aísla un número limitado de elementos para combinarlos y obtener un conjunto de tipos ideales que se pueden comparar con los casos reales. Define ocho tipos de sistemas de empresa, cuatro tipos de estados y seis tipos de sistemas de innovación. Este procedimiento analítico permite analizar aspectos específicos de la configuración institucional.

Una de las caracteristicas relevantes del análisis de Whitley es que pone de relieve que, a pesar de la fuerte heterogeneidad entre las regiones, el nivel nacional sigue siendo el más importante para el análisis de los arreglos institucionales, debido al papel central que los estadosnación siguen teniendo en el gobierno y regulación de la economía. Los sistemas empresariales son analizadas de acuerdo con el nivel de integración de la propiedad (los tipos que distingue son los siguientes: la fragmentaria, la de proyecto en red, la coordinada en forma de distrito industrial, y la forma de conglomerado, distinguiendo a su vez en esta última entre conglomerado financiero, integrado, compartimentado, de colaboración y altamente colaborativo).

Por otra parte, la coexistencia de sistemas empresariales en diferentes proporciones origina diferentes tipos de Estado. Los tipos ideales son: el de independencia absoluta, el de desarrollo dominante, el de espíritu empresarial corporativo y el corporativista inclusivo. Esta disposición es coherente con la propuesta de H\&S, donde la noción de complementariedad es también fundamental para la coherencia y la estabilidad de los tipos ideales, basados en los principios de eficiencia económica y el ejercicio del poder.

Estos diferentes tipos de estado están asociados con las caracteristicas de las empresas y los sistemas empresariales, 
a través de las estructuras de gobierno, las relaciones entre las empresas, las relaciones de trabajo y organización.

En lo referido a los sistemas de innovación, se definen por un número limitado de elementos (la división de la autoridad, la participación en los sistemas públicos de investigación, el grado de coordinación autoritarios, las innovaciones específicas del negocio, las innovaciones y la discontinuidad de la naturaleza sistémica de las innovaciones) que dan lugar a seis sistemas: los llamados sistemas autárquico, artesanal, de equipos de tecnología, dirigido por el Estado y de grupos de colaboración. Estos tipos de sistemas de innovación son más característicos de ciertos tipos de estado. Por ejemplo, el tipo autárquico y el tipo de equipos de tecnología son más comunes en los estados de independencia absoluta, mientras que los sistemas basados en grupos de colaboración existen generalmente en el estado corporativista.

Una cuestión central para Whitley (2003) es el papel de la ciencia en el apoyo al crecimiento de nuevas industrias. Tecnologías radicalmente innovadoras han variado entre países que fomentan diferentes niveles de la competencia, de reputación y el pluralismo intelectual y de flexibilidad (Tabla III). Estas dos características de los sistemas públicos de investigación ayudan a explicar las siguientes dimensiones: (i) las diferencias en el grado en que la investigación es coordinada a través de universidades y organizaciones semejantes para resolver problemas comunes, y (ii) la facilidad con que las nuevas metas y procedimientos se desenvuelven e incorporan en los programas de investigación para intentar resolver nuevos tipos de problemas. Este autor entiende un sistema público de investigación como aquel conjunto de entidades cuyos recursos humanos son asignados en mayor medida a la investigación, así como por los arreglos institucionales que dan forma a la financiación, la gestión y la evaluación de la ciencia.

Estas características de los sistemas públicos de investigación ayudan a entender cómo la producción de innovaciones y las empresas afrontan una variedad de problemas. Los sistemas públicos de investigación son, a su vez, afectados por cuatro características de los marcos institucionales que rigen la producción de conocimientos en los distintos países: i) la medida en que el estado delega en las élites intelectuales aquellas decisiones referidas al empleo y el control de los recursos, ii) la concentración del control administrativo dentro de las organizaciones de investigación, iii) la estabilidad y la fuerza de la jerarquía de las organizaciones de investigación, y iv) la segmentación de los objetivos de la organizaciones en el trabajo de investigación que realizan. En conjunto, estas características ayudan a explicar las grandes diferencias en la competencia científica y el pluralismo entre los sistemas.

El Estado promueve la diversidad de las relación existentes entre la empresas y la universidad (Whitley, 2007b). Las universidades se ven afectadas por una influencia que es especialmente relevante, dado que es el actor central de los sistemas públicos de investigación: se trata de la forma en que las universidades adquieren y utilizan los recursos, de la dirección de sus actividades básicas y del desarrollo de su capacidad organizativa. En sistemas de investigación altamente concentrados y jerarquizados los investigadores

TABLA III. TIPOS DE LOS SISTEMAS PÚBLICOS DE LA CIENCIA

\begin{tabular}{|l|l|l|}
\hline \multirow{2}{*}{$\begin{array}{c}\text { Nivel de pluralismo } \\
\text { y flexibilidad }\end{array}$} & \multicolumn{2}{|c|}{ Intensidad de Competencia por la Reputación } \\
\cline { 2 - 4 } Baja & $\begin{array}{l}|c| \\
\text { Beja } \\
\text { intelectuales de carácter incremental en programas central- } \\
\text { mente planeados }\end{array}$ & $\begin{array}{l}\text { Jerarquias competitivas: potenciación de contribuciones para } \\
\text { objetivos disciplinares insertados en cuadros establecidos }\end{array}$ \\
\hline Elevada & $\begin{array}{l}\text { Pluralismo diferenciado: sistemas que potencian diversos } \\
\text { programas en diferentes organizaciones, en diferentes pro- } \\
\text { blemas }\end{array}$ & $\begin{array}{l}\text { Pluralismo Competitivo: sistemas que potencian riesgo in- } \\
\text { telectual y abordajes variadas y mutables en problemas } \\
\text { comunes }\end{array}$ \\
\hline
\end{tabular}

Fuente: Whitley (2003: 1019). 
tienden a permanecer en la misma universidad, existiendo escasa movilidad entre varias organizaciones, lo que dificulta la transferencia de conocimiento. Un sistema de investigación más flexible facilita la participación plural de negocios en la investigación. La participación de las empresas en la investigación puede ser pasiva e indirecta (sobre todo con la absorción de personal cualificado) 0 activa (con lazos de colaboración intensos para el estudio de los problemas genéricos).

\section{Corolario 2}

Whitley, con el análisis de los sistemas públicos de investigación, pone de relieve cómo la flexibilidad y la capacidad para adquirir, desarrollar y utilizar nuevos conocimientos se ve facilitada en mayor o menor medida por algunos arreglos institucionales. Aunque la empresa sigue siendo el agente cuya conducta establece el conjunto de la economía, las organizaciones universitarias se consideran actores centrales en el sistema de investigación. Los modos en que las actividades innovadoras se organizan en los países dependen del dominio de las transacciones anónimas del mercado, o bien de las relaciones de cooperación reguladas por compromisos que adoptan los gobiernos. Whitley sugiere que compartir conocimientos y colaborar en innovación tiene menos riesgos en los contextos últimos. No obstante, esto puede, como sugieren también $H \& S$, inhibir la aparición de innovaciones radicales, que son discontinuas (o disruptivas) en relación con el conjunto de conocimientos y tecnología de las comunidades involucradas. A pesar de ello, la aproximación de Whitley supone una implicación muy importante para las economías coordinadas: también pueden ser competitivas en sectores emergentes de tecnología si adoptan estrategias de nicho en sus empresas, y si se adecúan a las ventajas comparativas institucionales en estos sectores. La transferencia de conocimiento es relativamente lenta entre las organizaciones en los sistemas públicos de investigación altamente segmentados en términos de objetivos, carrera profesional y recursos. En ellos hay dificultades para responder a los resultados de nuevas investigaciones si las trayectorias individuales permanecen aisladas. La existencia de objetivos comunes, ya sea en la investigación fundamental o aplicada, es esencial como catalizador de la transferencia. Por ello, las fronteras entre la investigación básica y la producción de conocimiento orientado a la práctica resultan más fluidas y permeables en los sis- temas orientados a la resolución de problemas prácticos. Esto facilita la integración de las empresas en redes de investigación, en comparación con los sistemas en que existe una división disciplinaria más marcada.

\subsection{La gobernanza y los sistemas sociales de innovación y producción}

El enfoque de la gobernanza es un macro-análisis que busca comprender y clasificar los mecanismos de coordinación del gobierno de la actividad económica mediante varias ampliaciones de los enfoques. Hollingsworth y Boyer (1997) muestran claramente cómo no todas las relaciones sociales se basan en el mercado. Existen mecanismos de coordinación que proporcionan a los actores vocabularios y lógicas para la consecución de sus objetivos y para la definición de lo que debe valorarse, mostrando las reglas y leyes que deben seguir en una determinada situación. Estos mecanismos de coordinación están asociados con "sistemas sociales de producción" que dan lugar a diferentes resultados económicos.

Un sistema social de producción se entiende como el conjunto de instituciones y estructuras de un país o región que se integran en una configuración social distintiva. Está formado por los siguientes subsistemas: el sistema de relaciones laborales, el sistema de formación para los trabajadores y directivos, el gobierno corporativo, las relaciones entre las empresas, las relaciones comerciales con proveedores y clientes, los mercados financieros, las concepciones de la justicia e equidad en los mercados de capital y de trabajo, y la estructura y política del estado. Todas estas instituciones, organizaciones y valores sociales tienden a ser consistentes.

Según Boyer (2003), un análisis económico de las instituciones debe tener en cuenta varios componentes: el habitus, las convenciones, la organización, las reglas institucionales y el orden constitucional. Boyer $(2003,2004)$ distingue cinco formas institucionales básicas, que podemos llamar building blocks: el régimen de la moneda, las relaciones salariales, la competencia, la adhesión al régimen internacional y el Estado. El régimen de acumulación es el conjunto de regularidades que garantizan un proceso global relativamente coherente de la acumulación de capital, lo que puede aumentar o reducir las distorsiones y los desequilibrios que resultan de estos procesos. 
El modo de regulación comprende un conjunto de procedimientos y comportamientos, tanto individuales como colectivos, que reproducen históricamente las relaciones sociales fundamentales a través de la conjunción de ciertas formas institucionales que provienen de una trayectoria histórica, lo que permite mantener el régimen de acumulación vigente y garantizar la dinámica de las decisiones descentralizadas.

Amable y Petit (1999) se refieren a las dimensiones institucionales que son cruciales para la comprensión de diversos mecanismos económicos y discuten el concepto de la arquitectura institucional para ilustrar cómo las instituciones pueden formar una estructura coherente y complementaria. Dada la diversidad institucional, estos autores sugieren dos enfoques distintos para las instituciones relacionadas con cada dimensión específica a analizar. El primer enfoque se basa en la idea de que el análisis de todas las actividades relacionadas con un tema particular nos permite comprender las instituciones centrales. Según los autores, este método puede no ser tan eficaz, porque en la práctica no existe una coincidencia exacta entre las actividades y las instituciones relacionadas. Éste es el enfoque típico, por ejemplo, en muchos estudios que utilizan el paradigma del sistema nacional de innovación. Un segundo enfoque, que adopta una visión más amplia proveniente de la escuela francesa de la Regulación, utiliza la noción de complementariedad institucional. Los autores toman una noción más comprehensiva, distinguiendo tres tipos:

- Tipo 0: Cuando las instituciones son inducidas por actividades conexas.
- Tipo 1: Cuando dos o más instituciones se refuerzan mutuamente y tienen efectos conjuntos sobre las actividades y los actores.

- Tipo 2: Cuando una institución tiene un efecto en otra institución.

La noción de los sistemas sociales de la innovación y la producción (SSIP), de Amable, Barré y Boyer (1997), son por tanto un intento de superar la visión limitada de los sistemas de innovación, que normalmente se presentan como un subconjunto del total de la economía. El análisis SSIP revela cómo el núcleo de un sistema de innovación, que consiste en el triángulo de la ciencia y la tecnología, la innovación y la industria, que es en gran medida interdependiente de tres vértices esenciales: la educación/capacitación, los recursos humanos y el sistema financiero. Las características que constituyen el sistema social de la innovación en cada territorio tienen un impacto en las capacidades económicas, generando las especificidades del sistema productivo.

Utilizando datos recientes, Amable y Lung (2008) analizan variables de cinco componentes esenciales: [1] la competencia de los productos en el mercado, [2] la negociación salarial y el mercado de trabajo, [3] la intermediación financiera y el gobierno empresarial, [4] la protección social, y [5] el sector de la educación. A partir de estas cinco variables identifican cuatro modelos principales de sistemas de innovación y producción en la Unión Europea: las economías de mercado (economía liberal de mercado o modelo anglo-sajón), las economías socialdemócratas, el capitalismo de Europa continental y el capitalismo en el Sur de Europa (Mediterráneo) (Ver Tabla IV).

TABLA IV. LOS CUATRO TIPOS DE CAPITALISMO EN EUROPA

\begin{tabular}{|c|c|c|c|c|}
\hline SSIP & $\begin{array}{c}\text { Economías basadas } \\
\text { en el mercado }\end{array}$ & $\begin{array}{c}\text { Economías } \\
\text { socialdemócratas }\end{array}$ & $\begin{array}{c}\text { Capitalismo europeo } \\
\text { continental }\end{array}$ & $\begin{array}{c}\text { Capitalismo del sur } \\
\text { de Europa }\end{array}$ \\
\hline $\begin{array}{l}\text { Competencia } \\
\text { del producto } \\
\text { en el mercado }\end{array}$ & $\begin{array}{l}\text { Importancia de la competen- } \\
\text { cia de precios. Neutralidad } \\
\text { del Estado en los mercados } \\
\text { de productos. Apertura a la } \\
\text { inversión extranjera y la com- } \\
\text { petencia internacional. }\end{array}$ & $\begin{array}{l}\text { Importancia de la competencia } \\
\text { por la calidad. Fuerte interven- } \\
\text { ción estatal en los mercados. } \\
\text { Alto grado de coordinación. } \\
\text { Apertura a la competencia y } \\
\text { la inversión extranjera. }\end{array}$ & $\begin{array}{l}\text { Importancia moderada en el } \\
\text { precio, relativamente más altos } \\
\text { debido a la calidad. Participa- } \\
\text { ción de las autoridades públi- } \\
\text { cas. Coordinación relativamen- } \\
\text { te alta por mecanismos distintos } \\
\text { al precio. Escasa protección } \\
\text { contra las empresas y la inver- } \\
\text { sión extranjera. }\end{array}$ & $\begin{array}{l}\text { Competencia de precios. Par- } \\
\text { ticipación del Estado en la } \\
\text { coordinación por mecanismos } \\
\text { distintos al precio. Protección } \\
\text { moderada contra las empre- } \\
\text { sas y la inversión extranjera. } \\
\text { Importancia de las pequeñas } \\
\text { empresas. }\end{array}$ \\
\hline
\end{tabular}


TABLA IV. LOS CUATRO TIPOS DE CAPITALISMO EN EUROPA (continuación)

\begin{tabular}{|c|c|c|c|c|}
\hline SSIP & $\begin{array}{c}\text { Economías basadas } \\
\text { en el mercado }\end{array}$ & $\begin{array}{c}\text { Economías } \\
\text { socialdemócratas }\end{array}$ & $\begin{array}{c}\text { Capitalismo europeo } \\
\text { continental }\end{array}$ & $\begin{array}{c}\text { Capitalismo del sur } \\
\text { de Europa }\end{array}$ \\
\hline $\begin{array}{l}\text { Negociación salarial } \\
\text { y mercado de } \\
\text { trabajo }\end{array}$ & $\begin{array}{l}\text { Débil protección del empleo. } \\
\text { Flexibilidad externa. Recurso } \\
\text { fácil al empleo temporal. } \\
\text { Contratación fácil. Política } \\
\text { de empleo activa. Tácticas } \\
\text { de defensa de los sindicatos. } \\
\text { Descentralización de la nego- } \\
\text { ciación salarial. }\end{array}$ & $\begin{array}{l}\text { Protección del empleo mode- } \\
\text { rada. Coordinación por nego- } \\
\text { ciación centralizada. Políticas } \\
\text { activas de empleo. Sindicatos } \\
\text { fuertes. Relaciones de coope- } \\
\text { ración industrial. }\end{array}$ & $\begin{array}{l}\text { Protección del empleo fuerte. } \\
\text { Flexibilidad externa limitada. } \\
\text { Estabilidad laboral. Conflictos } \\
\text { en las relaciones laborales. } \\
\text { Política de empleo activa. Sin- } \\
\text { dicatos moderadamente fuer- } \\
\text { tes. Negociación salarial coor- } \\
\text { dinada. }\end{array}$ & $\begin{array}{l}\text { Franja de empleo "flexi- } \\
\text { ble" de trabajo temporal y } \\
\text { a tiempo parcial. Posibles } \\
\text { conflictos en las relaciones } \\
\text { laborales. Política de empleo } \\
\text { activa. Centralización de la } \\
\text { negociación salarial. }\end{array}$ \\
\hline $\begin{array}{l}\text { Intermediación } \\
\text { financiera y } \\
\text { gobierno corporativo }\end{array}$ & $\begin{array}{l}\text { Sólida protección de los ac- } \\
\text { cionistas minoritarios. Falta } \\
\text { de concentración de la pro- } \\
\text { piedad. Grandes inversores } \\
\text { institucionales. Mercado acti- } \\
\text { vo para el control de la em- } \\
\text { presa (control de precios, fu- } \\
\text { siones y adquisiciones). Alta } \\
\text { sofisticación de los mercados } \\
\text { financieros. Desarrollo de ca- } \\
\text { pital de riesgo. }\end{array}$ & $\begin{array}{l}\text { Fuerte concentración de la } \\
\text { propiedad. Importancia de } \\
\text { los inversores institucionales. } \\
\text { No existe un mercado para } \\
\text { el control de las empresas } \\
\text { (control de precios, fusiones } \\
\text { y adquisiciones). Sofisticación } \\
\text { de los mercados financieros. } \\
\text { Alto grado de concentración } \\
\text { bancaria. }\end{array}$ & $\begin{array}{l}\text { Débil protección de los accio- } \\
\text { nistas externos. Concentración } \\
\text { de propiedad. No hay mercado } \\
\text { activo para el control de la } \\
\text { empresa (adquisiciones, fusio- } \\
\text { nes y adquisiciones). Baja so- } \\
\text { fisticación de los mercados } \\
\text { financieros. Desarrollo de ca- } \\
\text { pital de riesgo moderado. } \\
\text { Fuerte concentración banca- } \\
\text { ria. Importancia de los bancos } \\
\text { de Negocios y Finanzas. }\end{array}$ & $\begin{array}{l}\text { Débil protección de los ac- } \\
\text { cionistas externos. Fuerte } \\
\text { concentración de la pro- } \\
\text { piedad. Gestión empresarial } \\
\text { basado en los bancos. Algún } \\
\text { mercado activo para el con- } \\
\text { trol de la empresa (adqui- } \\
\text { siciones, fusiones y adqui- } \\
\text { siciones). Baja sofisticación } \\
\text { de los mercados financieros. } \\
\text { Desarrollo limitado de capi- } \\
\text { tal de riesgo. Alta concen- } \\
\text { tración bancaria. }\end{array}$ \\
\hline Protección social & $\begin{array}{l}\text { Protección social deficien- } \\
\text { te. Escasa participación del } \\
\text { Estado, centrándose en la } \\
\text { lucha contra la pobreza. } \\
\text { Prestaciones condicionadas a } \\
\text { los recursos. Sistemas de pen- } \\
\text { siones por capitalización. }\end{array}$ & $\begin{array}{l}\text { Alto nivel de protección social. } \\
\text { Propiedad del gobierno fuerte. } \\
\text { Importancia de la protección } \\
\text { social para la formulación de } \\
\text { políticas públicas y la socie- } \\
\text { dad. }\end{array}$ & $\begin{array}{l}\text { Alto grado de protección so- } \\
\text { cial. Protección social basada } \\
\text { en la participación del Estado } \\
\text { en el empleo. Importancia } \\
\text { de la protección social en la } \\
\text { sociedad. Financiación de se- } \\
\text { guridad social por contribu- } \\
\text { ciones. Sistema de pensiones } \\
\text { contributivas. }\end{array}$ & $\begin{array}{l}\text { Nivel moderado de la es- } \\
\text { tructura de los gastos de } \\
\text { protección social, orientados } \\
\text { a combatir la pobreza y las } \\
\text { jubilaciones. Fuerte inter- } \\
\text { vención estatal. }\end{array}$ \\
\hline Educación & $\begin{array}{l}\text { Débil gasto público. Sistema } \\
\text { de educación superior alta- } \\
\text { mente competitivo. Educación } \\
\text { secundaria no homogeneizada. } \\
\text { Baja formación profesional, } \\
\text { centrándose en las habilida- } \\
\text { des generales. Formación a lo } \\
\text { largo de la vida. }\end{array}$ & $\begin{array}{l}\text { Alto nivel de gasto público. } \\
\text { Alta tasa de matriculación. } \\
\text { Importancia de la calidad de } \\
\text { la educación primaria y se- } \\
\text { cundaria. Importancia de la } \\
\text { formación del profesorado, } \\
\text { centrado en competencias es- } \\
\text { pecíficas. Importancia de la } \\
\text { educación permanente. }\end{array}$ & $\begin{array}{l}\text { Alto nivel de gasto público. } \\
\text { Altas tasas de matriculación } \\
\text { en la enseñanza secundaria. } \\
\text { Énfasis en la homogeneidad } \\
\text { de la educación secundaria. } \\
\text { Formación profesional centra- } \\
\text { da en habilidades específicas. }\end{array}$ & $\begin{array}{l}\text { Gasto público débil. Tasas de } \\
\text { matrícula débil en la edu- } \\
\text { cación superior. Sistema de } \\
\text { educación superior de for- } \\
\text { mación frágil. Débil servicio } \\
\text { de formación, centrado en } \\
\text { las habilidades generales. }\end{array}$ \\
\hline
\end{tabular}

Fuente: Amable (2005), adaptado por el autor: 


\section{Corolario 3}

El enfoque de la gobernanza, del cual los SSIP son un ejemplo, es un análisis institucional comparativo, vinculado de manera importante a un componente empírico, incorporando en la investigación variables cuantitativas relevantes. Mientras que las ramas anteriores de las variedades del capitalismo se centraban en la empresa institucionalmente "incrustada" como elemento central y punto de partida del análisis, este enfoque destaca en cómo el conjunto de contextos institucionales influyen en las macro-variables que se reflejan en los indicadores estadísticos. La empresa deja de ser el foco del análisis para compartir el interés con otros actores como las universidades, que tienen un papel central en los diferentes sub-sistemas que constituyen el sistema social de la innovación y de la producción de un pais. El sistema de relaciones institucionales entre estos dominios se lleva a cabo a través de un concepto de complementariedad institucional enriquecido, que adicionalmente ofrece paralelismos con la idea del análisis de componentes principales empleada en varios procedimientos estadísticos multivariantes, lo cual supone una ventaja para hacer operativo el enfoque.

\section{IMPLICACIONES Y LIMITACIONES DE LOS ENFOQUES SOBRE LAS VARIEDADES DE CAPITALISMO}

La transferencia de conocimiento se asume como un proceso central para la innovación y el desarrollo. La manera de entender el vínculo entre la universidad y la industria se ha transformado para convertirse en una red de conocimientos territorialmente integrada. Se trata de un proceso caracterizado por diferentes mecanismos que son comunes en relación a los contextos específicos donde los actores participan en la transferencia. La arquitectura institucional restringe o mejora las actividades y comportamientos de los actores. En este sentido, las instituciones deben ser un aspecto central en la comprensión de cómo las universidades están interrelacionados con el entorno, en particular con las empresas, que son las que necesitan en distinto grado conocimientos para llevar al mercado. Las diferencias entre los territorios no sólo crean variaciones en los resultados económicos, sino también en la gobernanza. La configuración y la intensidad de uso de varios mecanismos de transferencia de conocimiento dependen, por tanto, de la arquitectura institucional. Por ejemplo, el uso de las patentes como herramienta central de la transferencia de conocimiento se lleva a cabo, entre otras cosas, debido a los marcos jurídicos de los distintos países que dan atención diferente a la protección de los derechos de propiedad. En los EE.UU. o el Reino Unido, la vigilancia sobre este tema es fuerte, por lo que la relación entre la universidad y la empresa asume en mayor medida este mecanismo de transferencia, estimulando las actividades de concesión de licencias. Al contrario, en las economías mediterráneas, que de acuerdo con Teece (1986) son caracterizadas como de un sistema débil de propiedad, es difícil proteger los derechos de propiedad de los conocimientos y la tecnología, por lo que las patentes tienen dificultades para ser consideradas como un mecanismo útil de la relación entre la universidad y empresa. Más bien se utilizan como un indicador de la capacidad tecnológica y como una expresión de la voluntad para transferir los conocimientos científicos.

Los distintos enfoques analizados ofrecen importantes contribuciones al análisis institucional de la transferencia de conocimiento, enfatizando cómo las instituciones se organizan y complementan, creando lógicas de articulación que estructuran las reglas del juego. No obstante, la perspectiva de las variedades del capitalismo no está exenta de críticas.

El punto de vista de H\&S ha sido ampliamente criticado debido a la forma en que se realiza la conexión entre la dinámica de la innovación y la coordinación de las economías. Taylor (2004) fue uno de los primeros críticos de las economías de especialización en innovación con diferentes grados de radicalismo. Este autor argumenta que los EE.UU. son un caso extremo y no pueden ser tomados como un ejemplo de base para esta comparación. Parte muy significativa de la innovación en el mundo de hoy es financiada y ejecutada por el Estado y las universidades. Las relaciones internacionales son también un aspecto que no debe pasarse por alto, debido a que la dinámica raramente es totalmente endógena, sino que depende en gran medida de las relaciones de los sistemas de investigación con otros sistemas externos. Por su parte, Akkermans et al. (2009) ha apoyado las criticas de Taylor, sobre todo debido a las debilidades de la evidencia empírica aportada por H\&S. Defender una tesis de carácter general basada en una prueba con sólo dos países (EE.UU. y Alemania), 
donde además se pasa por alto el distinto grado en que las innovaciones son radicales, resulta problemático para generalizar el análisis. No obstante, con el uso de un análisis longitudinal de varios países de la OCDE, estos autores han utilizado una amplia gama de indicadores de innovaciones radicales, realizando una serie de comparaciones con las que concluyen que, aunque el corolario de H\&S no puede ser tomado como una regla general, puede explicar una parte significativa de la conducta de las industrias en diferentes países.

Whitley también subraya, al igual que H\&S, el papel central del Estado en la estructuración de los sistemas empresariales. Los Estados, al interferir en la economía, causan una gran diversificación de arquitecturas institucionales. Las formas en que se organizan las actividades innovadoras en los paises dependen en gran medida de que dominen las transacciones de mercado, o bien de que existan relaciones de cooperación gobernadas por compromisos que son posibles gracias a las autoridades que organizan la interacción entre los actores. Un grado más bajo de autoridad y coordinación provoca falta de voluntad para establecer compromisos de largo plazo y para fomentar el aprendizaje entre organizaciones. Whitley sugiere que compartir conocimientos y colaborar en la innovación es menos arriesgado en estos contextos, aunque ello puede inhibir la aparición de innovaciones radicales discontinuas (o disruptivas) frente a contextos donde el conocimiento y la tecnología son desarrollados por las comunidades involucradas. Sin embargo, las economías coordinadas también pueden alcanzar la competitividad en sectores tecnológicos emergentes, al centrarse en estrategias de nicho cuando las empresas obtienen ventajas comparativas institucionales. Esta idea contradice la suposición de que el potencial de innovación europeo se limita a sectores tecnológicos de media intensidad.

La visión difundida por Amable ofrece un enfoque sistemático en muchas dimensiones institucionalmente relevantes, donde se unen los datos macroeconómicos de una forma consistente con los supuestos de los enfoques anteriores que se centran en la empresa. La idea del sistema social de la innovación y la producción puede ser muy interesante como punto de partida para el análisis, anclado en datos empíricos, de las tipologías de los países en aspectos vinculados a la innovación. Un claro contras- te entre este enfoque y los dos anteriores es que el énfasis en la empresa se sustituye por una visión integrada del sistema que afecta a la gobernanza de la innovación y la producción. El marco institucional de la transferencia de conocimiento está estrechamente relacionado con la complementariedad institucional que se refleja en los comportamientos de los actores y la influencia de las actividades económicas y las actuaciones de los territorios. Amable extiende las variedades de capitalismo de H\&S para crear tipos complejos que tienen un mayor alcance. El problema radica en el hecho de que, en efecto, cada estado-nación tiene un tipo único de capitalismo, con instituciones especificas. La idea original de H\&S sigue siendo muy atractiva por su simplicidad conceptual que da lugar a dos tipos ideales que contrastan los componentes esenciales de la arquitectura institucional de un pais.

Una limitación importante de los enfoques descritos es la dificultad para analizar escalas subnacionales, donde las cuestiones de proximidad y el aprendizaje son esenciales, algo que es considerado como el paradigma actual de los sistemas regionales de innovación (Carrincazeaux, Lung y Vicente, 2008; Gossling y Rutten, 2007; Asheim y Gertler, 2004; Asheim y Isaksen, 2002; Cooke, 1998). Varios autores han hecho un esfuerzo para conciliar los dos enfoques. Gambarotto y Solari (2009) pusieron a prueba las diferencias regionales en la UE en términos de las variedades de capitalismo. Con este fin, teniendo por punto de partida la tipología de Amable, han encontrado evidencia de las diferencias regionales relacionadas con los diferentes perfiles institucionales. También Carrincazeaux y Gaschet (2006) tratan de entender los perfiles regionales con una batería de indicadores relacionados con las dimensiones sugeridas, dirigido a la construcción de tipologías de las regiones europeas en términos de configuración de la ciencia, la tecnología y la industria. Los resultados ilustran una amplia gama de perfiles regionales, donde subsisten regiones que tienen una fuerte diversidad en términos económicos. Los autores enfatizan que, a pesar de esta diversidad, la dimensión nacional sigue dominando las diferencias en el rendimiento de las economías regionales. Ésta es una conclusión relevante de la literatura sobre variedades de capitalismo, donde el nivel nacional sigue siendo apropiado para una comparación institucional y resulta esencial debido al papel crucial de los estados nacionales en la regulación. 
La existencia de formas de coordinación en las economías que van más allá que los mercados, tales como las redes o las comunidades, son fundamentales para el proceso de transferencia de conocimiento. Justifican por sí solas el gran interés que para el estudio de la transferencia de conocimiento tienen los marcos conceptuales de carácter institucionalista. En definitiva, el análisis detallado de la transferencia de conocimiento debe considerar como punto de partida la amplia gama de aspectos institucionales que crean las bases para la diferenciación de los comportamientos de los actores.

\section{NOTAS}

1 Una versión preliminar de este artículo en lengua portuguesa ha sido publicada en e-Cadernos CES, n. ${ }^{\circ}$, 2009 (www.ces.uc.pt/e-cadernos).

\section{REFERENCIAS BIBLIOGRÁFICAS}

Akkermans, D. et al. (2009), "Do 'liberal economies' really innovate more radically than 'coordinated market economies'? Hall and Soskice reconsidered", Research Policy, 38, Elsevier, 181-191.

Amable, B. (2005), Les cinq capitalismes: Diversité des systèmes économiques et sociaux dans la mondialisation, Paris: Seuil.

Amable, B. et al. (1997), Les Systèmes d'Innovation à l'Ére de la Globalisation, Paris: Economica.

Amable, B. y Lung, Y. (2008), The European Socio-Economic Models of a Knowledge-based Society. Main Findings and Conclusion, Cahiers du GREThA 200826, Groupe de Recherche en Economie Théorique et Appliquée.

Amable, B. y Petit, P. (1999), "Identifying the structure of institutions to promote innovation and growth", CEPREMAP Working Papers, 9919, disponible online en http://www.cepremap.cnrs. fr/couv_orange/co9919.pdf a 26-112009.

Aoki, M. (2001), Toward a Comparative Institutional Analysis, Cambridge y Londres: The MIT Press, 1-29.
Asheim, B. y Gertler, M. (2004), "The geography of innovation: Regional innovation systems" en Fagerberg J. et al. (eds.), The Oxford Handbook of Innovation, Oxford: Oxford University Press.

Asheim B. y Isaksen A. (2002), "Regional Innovation Systems: The Integration of Local 'Sticky' and Global 'Ubiquitous' Knowledge", Journal of Technology Transfer, 27, 77-86.

Bercovitz, J. y Feldman, M. (2005), "Entrepreneurial Universities and Technology Transfer: A Conceptual Framework for Understanding Knowledge-Based Economic Development", The Journal of Technology Transfer, Springer, 31, 175-188.

Berman, E. P. (2008), "Why Did Universities Start Patenting? Institution-Building and the Road to the Bayh-Dole Act", Social Studies of Science, 38.

Bok, D. (2003), Universities in the Marketplace - The Commercialization of Higher Education, New Jersey: Princeton University Press.

Boyer, R. (2004), Une théorie du capitalisme est-elle possible?, Paris: Odile Jacob.

Boyer, R. (2003), "Les institutions dans la théorie de la régulation", CEPREMAP Working Papers, n. 2003-08, disponible online em www.cepremap.cnrs. fr/couv_orange/co0308.pdf a 26-112009.

Bozeman, B. (2000), "Technology transfer and public policy: A review of research and theory", Research Policy, 29, Elsevier, 627-655.
Aceptado: 16 de junio de 2011 
Bromley, D. (2006), Sufficient Reason: Volitional Pragmatism and the Meaning of Economic Institutions, New Jersey: Princeton University Press.

Carrincazeaux, C. y Gaschet, F. (2006), "Knowledge and the Diversity of Innovation Systems: A Comparative Analysis of European Regions", Cahiers du GRES, 29, Université MontesquieuBordeaux IV.

Carrincazeaux, C.; Lung, Y. y Vicente, J. (2008), "The Scientific Trajectory of the French School of Proximity: Interaction and Institution-based Approaches to Regional Innovation Systems", European Planning Studies, 16, 617-628.

Casper, S. (2006), "Export the Silicon Valley to Europe: How Useful is Comparative Institutional Theory?", en Hager, J. y M. Meeus (eds.), Innovation, Science, and Institutional Change, New York: Oxford University Press, 483-504.

Casper, S. et al. (1999), "Can high-technology industries prosper in Germany: institutional frameworks and the evolution of the German software and biotechnology industries", Industry and Innovation, Routledge, 6, 6-23.

Cohen W. y Levinthal, D. (1990), "Absorptive capacity: a new perspective on learning and innovation", Administrative Science Quarterly, 35, 128-152.

Cooke, P. (1998), "Origins of the concept", en Braczyc, H. J. et al. (eds.), Regional Innovation Systems - The role of governances in a globalized world, 1. a ed., Londres: UCL Press.

David, P. (1994), Accessing and expanding the science and technology knowledge base, Working Group on Innovation and Technology Policy, Paris: OECD.

Debackere, K. y Veugelers, R. (2005), "The role of academic transfer organizations in improving industry science links", Research Policy, 34, Elsevier, 321-342.
D'Este, P. y Patel, P. (2007), "Universityindustry linkages in the UK: What are the factors underlying the variety of interactions with industry?", Research Policy, 36, Elsevier, 1295-1313.

Etzkowitz, H. (2003), "Research groups as 'quasi-firms': The invention of the entrepreneurial university", Research Policy, 32, Elsevier, 109-121.

Etzkowitz, H. y Leydesdorff L. (eds.) (1997), Universities and the Global Knowledge Economy - A Triple Helix of UniversityIndustry-Government Relations, Londres: Continuum.

Etzkowitz, H. et al. (2000), "The future of the university and the university of the future: Evolution of ivory tower to entrepreneurial paradigm", Research Policy, 29, Elsevier, 313-30.

European Commission (1995), Livro Verde sobre a Inovação, Bruxelas: CE.

Freeman, C. y Louçã, F. (2004), Ciclos e Crises no Capitalismo Global - Das Revoluções Industriais à Revolução da Informação, Porto: Edições Afrontamento.

Freeman, C. y Perez, C. (1988), "Structural crises of adjustment, business cycles and investment behaviour", en Dosi, G. et al. (eds.) (1988), Technical Change and Economic Theory, London: Pinter Publishers, 38-66.

Gibbons, M. et al. (1994), The New Production of Knowledge: The Dynamics of Science and Research in Contemporary Societies, Londres: Sage.

Goldstein, H. A. (2008), "The 'entrepreneurial turn' and regional economic development mission of universities", Annals of Regional Science, Springer.

Gossling, T. y Rutten, R. (2007), "Innovation in Regions", European Planning Studies, 15, 2, Taylor \& Francis, 253270.

Gunasekara, C. (2006), "Reframing the Role of Universities in the Development of Regional Innovation Systems", The
Journal of Technology Transfer, 31(1), Springer, 101-113.

Hall, P. A. y Soskice, D. (2001), "An Introduction to Varieties of Capitalism", en Peter A. Hall y David Soskice (eds.), Varieties of Capitalism: The institutional foundations of comparative advantage, 0xford: Oxford University Press, 1-68.

Hancké, B. (2009), "Introducing the Debate", en Hancké, B. (ed.) (2009), Debating the Varieties of Capitalism - A Reader, Oxford: Oxford University Press.

Hessels, L. y van Lente, H. (2008), "Rethinking new knowledge production: A literature review and a research agenda", Research Policy, 37, Elsevier, 740-760.

Hodgson, G. (2006), "What Are Institutions?", Journal of Economic Issues, $\mathrm{XL}$ (1), 1-25.

Hollingsworth, J. R. y Boyer, R. (1997), "Coordination of Economic Actors and Social Systems of Production", en J. Rogers Hollingsworth y Robert Boyer (eds.), Contemporary Capitalism: The Embeddedness of Institutions, Nueva York y Cambridge: Cambridge University Press.

Jackson, G. y Deeg, R. (2006), "How Many Varieties of Capitalism? Comparing the Comparative Institutional Analyses of capitalist Diversity", MPIG Discussion Paper, 06/2.

Latour, B. (1987), Science in Action: How to Follow Scientists and Engineers through Society, Cambridge Mass, USA: Harvard University Press.

Latour, B. (2005), Reassembling the Social, Oxford: Oxford University Press.

Molas-Gallart, J. et al. (2002), Measuring Third Stream Activities: Final Report to the Russell Group of Universities, Brighton: Science and Technology Policy Research Unit, University of Sussex. 
Nelson, R. (2008), "What enables rapid economic progress: What are the needed institutions?", Research Policy, 37, Elsevier, 1-11.

North, D. (1994), "Economics Performance Through Time", The American Economic Review, 8, 3, 359-368.

OECD (2005), Oslo Manual - Guidelines for Collecting and Interpreting Innovation Data, 3. ${ }^{a}$ ed., Paris: OECD Publications.

Phan, P. H. y Siegel, D. S. (2006), "The Effectiveness of University Technology Transfer: Lessons Learned from Qualitative and Quantitative Research in the U.S. and U.K", Rensselaer Working Papers in Economics, 06/09, Rensselaer Polytechnic Institute, Department of Economics.

Santos, B. S. (2008), "A Universidade no Século XXI: Para uma Reforma De- mocrática e Emancipatória da Universidade", en Santos, B. S. y Filho, N. (2008), A universidade no século XXI: Para uma universidade nova, Coimbra: Edições Almedina.

Solari, S. y Gambarotto, F. (2009), Regional Dispersion of Economic Activities and Models of Capitalism in Europe, Artigo de Apoio à Conferência no Programa de Doutoramento em Governação, Conhecimento e Inovação, Faculdade de Economia da Universidade de Coimbra, Fevereiro.

Taylor, M. Z. (2004), "Empirical Evidence Against Varieties of Capitalism's Theory of Technological Innovation", International Organization, 58, 601631.

Teece, D. J. (1986), "Profiting from technological innovation: Implications for integration, collaboration, licensing and public policy", Research Policy, 15, 285-305.

Whitley, R. (1999), Divergent Capitalisms: The Social Structuring and Change of Business Systems, Oxford: Oxford University Press.

Whitley, R. (2003), "Competition and pluralism in the public sciences: The impact of institutional frameworks on the organisation of academic science", Research Policy, 32, Elsevier, 1015-1029.

Whitley, R. (2007a), Business Systems and Organizational Capabilities - The Institutional Structuring of Competitive Competencies, Oxford: Oxford University Press.

Whitley, R. (2007b), Constructing universities as trategic actors: Limitations and varieties, Comunicación presentada en el Simposio "The Univesrity in the market", Estocolmo, 1-3 de noviembre. 